\title{
DESARROLLO DE UN ÍNDICE DE CONDICIÓN CORPORAL EN CUYES: RELACIONES ENTRE CONDICIÓN CORPORAL Y ESTIMADOS CUANTITATIVOS DE GRASA CORPORAL
}

\author{
Development of a Body Condition Score in Guinea Pigs: Relationships \\ Between Body Condition and Quantitative Estimates of Body Fat
}

\author{
Miguel Ara G. ${ }^{1,4}$, Ronald Jiménez A. ${ }^{3}$, Amparo Huamán C. ${ }^{3}$, Fernando Carcelén C. ${ }^{1}$, \\ Diego Díaz C. ${ }^{2}$
}

\section{RESUMEN}

El propósito del estudio fue contribuir al desarrollo y validación de un índice de condición corporal (ICC) para cuyes del Centro de Investigación IVITA, midiendo su consistencia entre evaluadores y su capacidad de predicción de la acumulación de grasa corporal. Se trabajó con 40 hembras Perú, las cuales fueron sometidas a restricciones variables en la dieta por cuatro semanas para obtener individuos representativos de cada unidad del ICC. Cuatro técnicos evaluaron el ICC de cada animal en una escala de 1 a 5 con incrementos de 0.5 ( 1 es emaciado, 5 es obeso). Luego, los cuyes fueron pesados y medidos para obtener el índice de masa corporal (IMC), y se beneficiaron para obtener la masa gravimétrica de los cuerpos grasos mesentérico, perirenal-uterino, dorso cervical caudal, mamario, poplíteo y axilar. Se estimó el grado de consistencia del ICC entre evaluadores mediante el coeficiente de correlación intraclase (CCI), la asociación entre el ICC y nueve estimados cuantitativos de grasa corporal mediante análisis de correlación, y la capacidad de predecir variaciones en los estimados cuantitativos mediante análisis de regresión. El ICC mostró buena consistencia entre evaluadores (CCI = 0.77), y una correlación significativa con todos los estimados de grasa corporal, siendo los coeficientes más altos con el IMC (0.87), grasa total (0.83) y grasa dorso cervical caudal (0.86). Cada incremento unitario en el ICC produce un incremento de $0.17 \pm 0.032$ en el IMC, $62.78 \pm 13.21 \mathrm{~g}$ en la grasa total y $8.03 \pm 1.46$ g en la grasa dorso cervical caudal. Los resultados indican que ICC en desarrollo es promisorio, tanto en su consistencia entre evaluadores como en la capacidad de predecir significativamente variaciones en grasa corporal.

Palabras clave: cuyes, condición corporal, masa corporal, grasa corporal, Mantaro, Perú

${ }^{1}$ Laboratorio de Bioquímica, Nutrición y Alimentación Animal, ${ }^{2}$ Clínica de Animales Menores, Facultad de Medicina Veterinaria, Universidad Nacional Mayor de San Marcos, Lima

${ }^{3}$ Estación Experimental del Centro de Investigación IVITA-El Mantaro, Huancayo

${ }^{4}$ E-mail: miguelara49@gmail.com 
The purpose of the study was to strengthen the development and validation of a body condition score (BCS) system for guinea pigs of the IVITA Research Centre, measuring its consistency among evaluators and its ability to predict fat changes. Forty Peru female guinea pigs were under feed restriction for four weeks to obtain animals in each unit of the BCS. Four technicians evaluated each animal in a 1 to 5 rating system, with 0.5 increments ( 1 = emaciated, 5 = obese). Then, the animals were weighed and measured to obtain the body mass index (BMI) and slaughtered to obtain the gravimetric mass of the mesenteric, perirenal-uterine, dorso-cervical-caudal, mammary, popliteal, and axilar fat deposits. The inter-rater reliability was assessed by the intraclass correlation coefficient (ICC); the association between BCS and nine quantitative estimates of body fat was measured through correlation analysis; and the ability to predict variations in the quantitative fat was measured through regression analysis. The BCS showed a good inter-rater reliability (CCI $=0.77)$, and its values were significantly correlated with all body fat estimates; however, the highest coefficients were obtained for IMC (0.87), total fat (0.83) and dorso-cervical-caudal fat (0.86). Each unit increment in BCS resulted in an increment of $0.17 \pm 0.032$ in BMI, $62.78 \pm 13.21 \mathrm{~g}$ in total fat, and $8.03 \pm 1.46 \mathrm{~g}$ in dorsocervical-caudal fat. These results suggest that the IVITA BCS system is a promissory management technique in terms of its consistency, good association with quantitative body fat and its ability to predict fat changes in guinea pigs.

Key words: guinea pigs, body condition, body mass, body fat, Mantaro, Peru

\section{INTRODUCCIÓN}

En un estudio sobre desarrollo de sistemas de crianza de cuyes implementado en la Sierra Central del Perú (INCAGRO, 2006), se obtuvieron resultados parciales que sugieren la posibilidad de incrementar la eficiencia reproductiva a través de la manipulación de la condición corporal de los reproductores en etapas críticas como el empadre y el inicio de la lactación. Asimismo, con el propósito de realizar una mejor transferencia de esta tecnología a los usuarios, el equipo de investigadores del Centro de Investigación IVITA de la Estación Experimental El Mantaro ha desarrollado un índice de condición corporal (ICC) aplicable a cuyes reproductores (Jiménez y Huamán, 2010).

Debido a su potencial de aplicación práctica, el ICC se ha convertido en una poderosa herramienta para el manejo productivo y reproductivo de vacunos de leche y carne (Hardin, 1990), ovinos (al-Sabbagh et al.,
1995), equinos (Gentry et al., 2004), cerdos (Esbenshade et al., 1986) e incluso mascotas (Dorsten y Cooper, 2004). El ICC se fundamenta en que la respuesta animal ante desafíos productivos y reproductivos depende más de la acumulación y movilización de reservas energéticas corporales que del mismo peso vivo (Garnsworthy, 1988).

A pesar de su amplio uso, el ICC no ha estado exento de críticas, principalmente en términos de su falta de precisión, subjetividad envuelta y de su posible falta de consistencia entre y dentro de evaluadores. Sin embargo, la capacidad predictiva del ICC ha sido razonablemente validada frente a métodos indirectos de estimación de grasa corporal en vacunos de leche (Domecq et al., 1995) y en yeguas (Gentry et al., 2004). Asimismo, sus categorías se encuentran significativamente asociadas a variaciones en la producción y reproducción en ovinos (Sanson et al., 1993) y vacunos de leche (Roche et al., 2007). Por otro lado, la consistencia del ICC entre evaluadores con variados niveles de 
entrenamiento ha sido demostrada en vacunos de leche (Edmonson et al., 1989).

No existen referencias publicadas sobre el desarrollo y aplicación de ICC para manejo de cuyes, a excepción de unos cuantos antecedentes en un contexto clínico (Shrubsole-Cockwill et al., 2008) o de folletos de extensión para el cuidado de mascotas (Veterinary Teaching Hospital, Michigan State University, 2011). Por otro lado, Aliaga et al. (2009) utilizan el término "buen estado corporal” para hembras próximas al parto; el cual, por su descripción, sería equivalente a la condición 3 del ICC de IVITA. Es el propósito de este estudio contribuir al desarrollo y validación del ICC para cuyes del Centro de Investigación IVITA - El Mantaro, midiendo su consistencia entre evaluadores y su capacidad de predicción de la acumulación de grasa corporal, a través del contraste con métodos directos como el índice de masa corporal (IMC) y la masa gravimétrica de diversos cuerpos grasos del animal.

\section{Materiales y MÉTOdos}

\section{Localización}

El estudio se llevó a cabo en la Unidad de Producción de Cuyes del Centro de Investigación IVITA - El Mantaro, localizada a $34 \mathrm{~km}$ de la ciudad de Huancayo, en la Sierra Central del Perú.

\section{El ICC}

Se trabajó con el ICC del IVITA descrito por Jiménez y Huamán (2010), donde cuatro técnicos de la Unidad de Producción de Cuyes fueron adiestrados en su determinación. El ICC en evaluación incluye las siguientes cinco categorías:

CC 1: Totalmente emaciado, con las vértebras cervicales, los apófisis de la vértebras torácicas y lumbares y la tuberosidad sacra completamente palpables.
CC 2: Mayor masa coporal pero aún son palpables todas las estructuras óseas mencionadas en CC 1.

CC 3: Ya no es posible palpar las vértebras cervicales ni las costillas, a menos que se ejerza mayor presión. Las demás estructuras óseas aún son palpables.

CC 4: Además de los mencionado en CC 3, ya no es posible palpar las apófisis de las vértebras torácicas ni lumbares. Aún es palpable la tuberosidad sacra, pero acompañada de tejido blando.

CC 5: Lo único que puede palparse levemente es la tuberosidad sacra. El cuello se presenta totalmente cilíndrico al tacto.

\section{Arreglo Experimental}

Se utilizaron 40 cuyes hembras vacías de un año de edad de la raza Perú. Los animales fueron identificados y distribuidos aleatoriamente en cinco grupos correspondientes a cada una de las categorías del ICC.

Las hembras pertenecientes a las condiciones 1 y 2 recibieron inicialmente forraje verde y paja de avena a discreción. Posteriormente, el forraje verde se fue restringiendo hasta 50 y 150 g por animal-día, respectivamente. Las hembras de la condición 3 recibieron paja de avena y afrechillo a discreción durante todo el periodo de alimentación. Las hembras de la condición 4 recibieron la misma dieta que las de condición 3 más harina de cebada a discreción. Las hembras de la condición 5 recibieron la dieta correspondiente a la condición 4 más harina de maíz, harina de haba y concentrado integral a discreción.

El tiempo máximo que les tomó a las hembras en aproximarse a la condición deseada fue de cuatro semanas, especialmente para las condiciones 4 y 5 .

\section{Aplicación del ICC y Estimación Cuan- titativa}

Una vez que las hembras se aproximaron sensiblemente a la CC correspondiente a 
su grupo experimental, los cuatro técnicos calificaron consecutiva (y ciegamente) los ICC de cada una de las 40 hembras en una escala de 1 a 5 , con incrementos de 0.5 .

Durante la calificación del ICC, se registraron los pesos y longitudes de los animales para el cálculo del índice de masa corporal (IMC) mediante la fórmula de peso/longitud $^{2}$ (Roubenoff et al., 1995). Inmediatamente después, los animales fueron sacrificados y se separaron quirúrgicamente los cuerpos grasos correspondientes a las zonas mesentérica, perirrenal-uterina, dorso cervical caudal, mamaria, poplítea y axilar, los cuales fueron pesados en una balanza con una precisión de $0.01 \mathrm{~g}$. Adicionalmente, se calculó la grasa total y su proporción respecto al peso vivo.

\section{Análisis Estadístico}

La consistencia en las calificaciones del ICC entre diferentes técnicos fue ilustrada mediante un diagrama de puntos y expresada mediante el coeficiente de correlación intraclase (CCI). El CCI fue calculado mediante un ANVA de dos vías, con animales y técnicos como factores aleatorios (Shrout y Fleiss, 1979) y su intervalo de confianza de $95 \%\left(\mathrm{IC}_{0.95}\right.$ ) fue aproximado usando el procedimiento descrito por Fleiss y Shrout (1978), asumiendo una distribución normal para los residuos del ANVA.

El grado y patrón de asociación entre las evaluaciones del ICC y los diferentes estimados cuantitativos de grasa corporal fueron calculados mediante análisis de correlación de Pearson en forma independiente. La capacidad de predicción del ICC para los valores grasos mejor correlacionados fue determinada mediante un modelo general lineal de la forma $y_{i}=b_{0}+b_{1} x_{1 i}+e_{i}$, donde $y$ es la expresión de grasa corporal, ya sea por el IMC o los cuerpos grasos, $x_{1 i}$ es la categoría de ICC y $e_{i}$ es el error aleatorio. El coeficiente de regresión $b_{1}$ es la variación en la grasa corporal por cada unidad de ICC.
Los cálculos estadísticos se efectuaron con la ayuda del paquete SAS ${ }^{\circledR} 9.2$ (SAS Institute Inc., 2009). Para todas las pruebas de hipótesis se usó un nivel de significación de 0.05 .

\section{REsultados y Discusión}

\section{Consistencia entre Evaluadores}

La distribución espacial de las calificaciones de ICC por cada evaluador y para cada animal se ilustra mediante un diagrama de puntos en la Fig. 1. Se observa una buena agregación alrededor de cada uno de los animales; esto es, la variabilidad en la calificación de los animales es mayor que la variabilidad que existe entre técnicos evaluadores en la calificación para un mismo animal. Esta tendencia es confirmada por el alto valor del CCI obtenido (0.77, $\left.\mathrm{IC}_{0.95}=0.62-0.87\right)$. Este coeficiente, interpretado como la proporción de la variabilidad debida a los animales, es considerado una buena medida de la consistencia entre evaluadores; donde usualmente el valor resultante es calificado como pobre $(<0.40)$, aceptable a bueno (0.40-0.75) y bueno (>0.75) (Haneline et al., 2009).

Si bien no existen experiencias publicadas sobre el grado de consistencia en la evaluación de ICC en cuyes, es posible comparar estos resultados con experiencias similares con otras especies. Nicoll (1979), trabajando con 41 bovinos calificados por dos evaluadores, obtuvo un coeficiente de correlación moderado (0.60) entre los registros de los evaluadores para el mismo animal. Igualmente, Fitzgerald et al. (2009) obtuvieron correlaciones de 0.59 y 0.58 usando ICC de 1 a 9 y de 1 a 5 , respectivamente, con 125 marranas y 17 evaluadores con diferente experiencia en la calificación de ICC. Mejores resultados fueron obtenidos por Audigé et al. (1998), quienes aplicaron el ICC en el venado rojo con dos evaluadores logrando coeficientes de 0.81 para las jóvenes $(n=39)$ y 0.79 para las adultas $(n=90)$. 


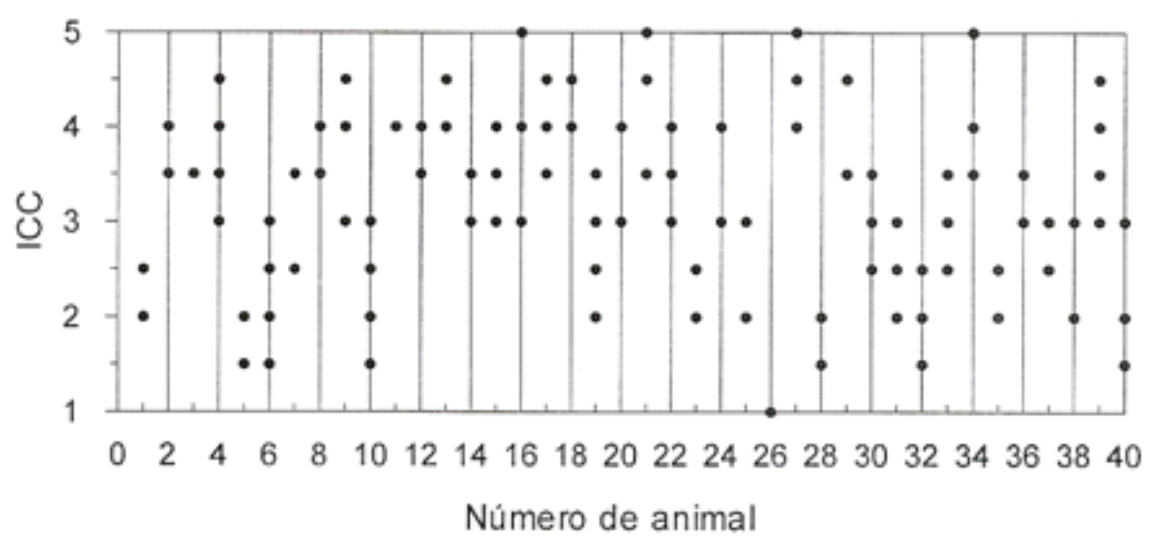

Figura 1. Diagrama de puntos de los índices de condición corporal (ICC) determinados por cuatro evaluadores en 40 cuyes hembra del Centro de Investigación IVITA El Mantaro, Junín. Obsérvese que los puntos dentro de animales están más aglomerados que entre animales. Aquellos animales con menos de cuatro puntos tienen sus ICC superpuestos. Coeficiente de correlación intraclase $=0.77$.

Es posible que algunas de las asunciones para el uso de ANOVA en la obtención del CCI hayan sido violadas, ya sea por la naturaleza discreta de los ICC, porque exista heterogeneidad de varianzas entre evaluadores, o porque la distribución de los ICC sea sesgada (en condiciones no experimentales es difícil obtener valores cercanos a los extremos de la escala). Estas posibilidades llevaron a Kristensen et al. (2006) a emplear el coeficiente de kappa ponderado [ $<0.4$ (deficiente), 0.4-0.8 (moderado) y $>0.8$ (excelente)], como alternativa al CCI para examinar la consistencia entre evaluadores del ICC en bovinos de leche, obteniendo coeficientes de 0.17 a 0.78 .

En el presente estudio, la elección del CCI, basado en un ANOVA, como expresión de consistencia es justificable considerando que los ICC finales fueron aproximados experimentalmente, los evaluadores tuvieron la misma experiencia y entrenamiento en la calificación de ICC, y la prueba de desviación de la normalidad para los residuos, después del efecto del evaluador y del animal (resultados no mostrados) no fue significativa.

\section{Asociación entre Variables}

Debido a la alta consistencia, el análisis de correlación entre los valores de ICC y los estimados cuantitativos de grasa corporal se hizo promediando los valores de ICC de los cuatro evaluadores. El Cuadro 1 muestra una alta y significativa correlación entre los ICC y el IMC, la grasa total y porcentual, y los cuerpos grasos mesentéricos, perirenal-uterino y dorso cervical caudal. El grado de correlación fue menor, aunque estadísticamente significativo, para los demás cuerpos grasos.

Correlaciones de ICC con estimados directos de grasa corporal han sido reportados por Sanson et al. (1993), empleando un ICC de 1 a 9 en ovejas y dos evaluadores entrenados, obteniendo coeficientes de correlación desde 0.84 con la masa combinada de los cuerpos grasos renal, pélvico y del corazón, hasta 0.97 con el porcentaje del grasa del cuerpo vacío (carcasa, órganos y vísceras limpias). Los ICC también han sido correlacionados con estimaciones indirectas de grasa corporal, especialmente aquellas obtenidas mediante ultrasonido. Así, Gentry et al. (2004), trabajando con yeguas y un ICC 

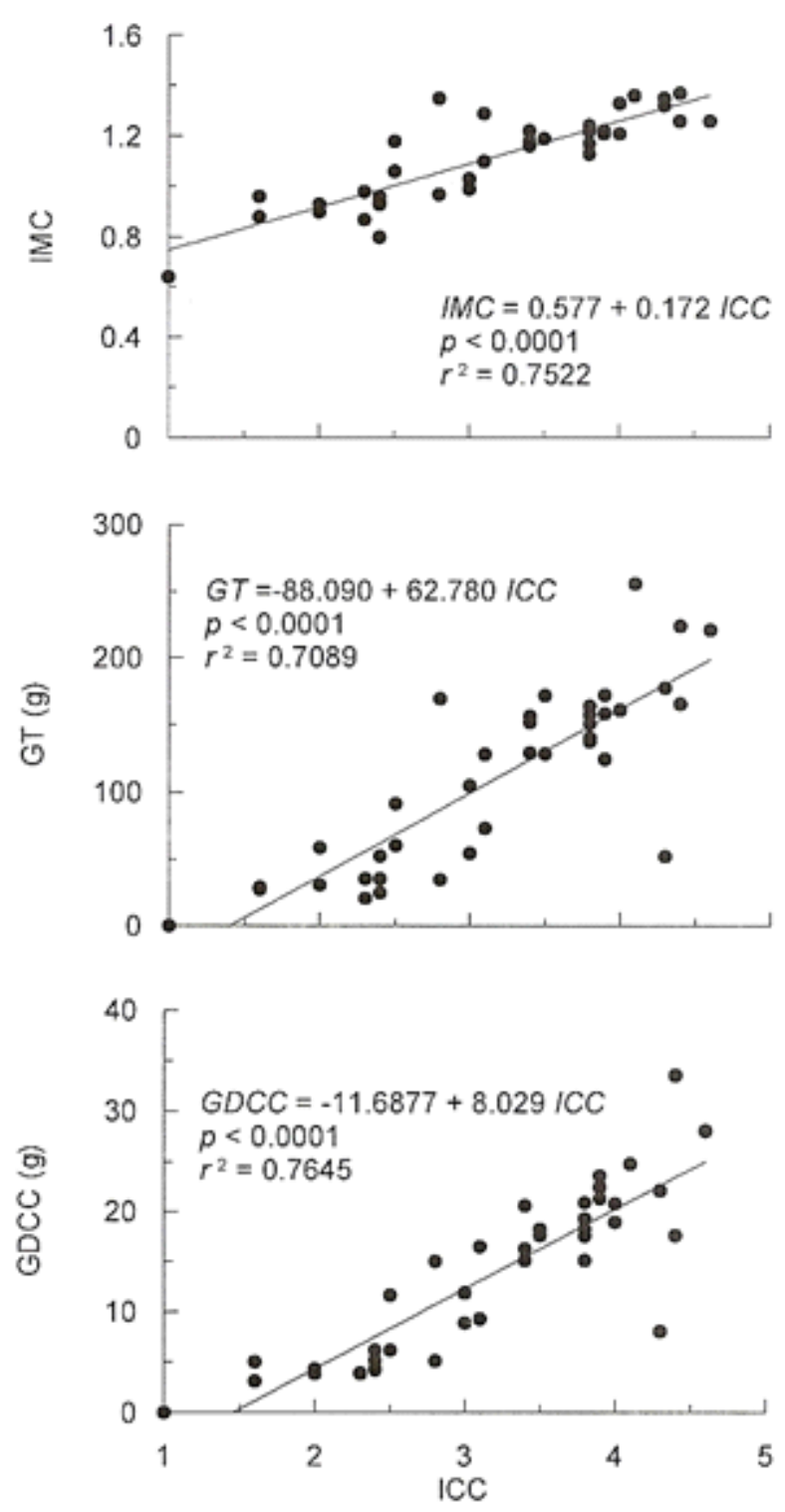

Figura 2. Regresiones lineales del índice de masa corporal (IMC), grasa total (GT) y grasa dorso-cervical-caudal (GDCC) sobre el índice de condición corporal (ICC) de 40 cuyes hembra del Centro de Investigación IVITA El Mantaro

de 1 a 9 , obtuvieron correlaciones de 0.87 , $0.84,0.82$ y 0.86 con el espesor de la grasa subcutánea en la base de la cola, el anca, la $13^{\mathrm{a}}$ costilla y la cruz, respectivamente. Similares correlaciones $(r=0.86)$ han sido obtenidas en búfalos con un ICC de 1 a 5 (Anitha et al., 2010). Es pertinente señalar que en todos estos casos, las condiciones corporales finales de los animales fueron generadas experimentalmente.

\section{Capacidad de Predicción de los ICC}

El análisis de regresión lineal mostró que los ICC promedio predijeron significativamente las variaciones en todos los estimados de grasa corporal (IMC y grasa mesentérica, perirrenal-uterina, dorso-cervical-caudal, mamaria, poplítea, axilar, total y porcentual). La Fig. 2 muestra los diagramas de dispersión del ICC y las ecuaciones de regresión 
Cuadro 1. Coeficientes de correlación ${ }^{1}$ entre los índices de condición corporal (ICC) y los estimados de grasa corporal de 40 cuyes hembras

\begin{tabular}{|c|c|}
\hline Estimado de grasa corporal & $r$ \\
\hline IMC & 0.87 \\
\hline Grasa total & 0.83 \\
\hline Grasa porcentual & 0.82 \\
\hline \multicolumn{2}{|l|}{ Cuerpos grasos } \\
\hline Mesentérico & 0.81 \\
\hline Peri-renal uterino & 0.83 \\
\hline Dorso cervical caudal & 0.86 \\
\hline Mamario & 0.70 \\
\hline Poplíteo & 0.61 \\
\hline Axilar & 0.74 \\
\hline
\end{tabular}

frente a las variables IMC, grasa total, y grasa dorso-cervical-caudal, las cuales fueron las que mostraron mejor correlación con el ICC. Las ecuaciones de regresión indican que cada incremento de una unidad en el ICC predice un incremento de $0.17 \pm 0.032$ en el IMC, de $62.78 \pm 13.21 \mathrm{~g}$ en la grasa total y de $8.03 \pm 1.46$ g en la grasa dorso-cervicalcaudal.

Los resultados permiten concluir que el índice de condición corporal que se viene desarrollando en el Centro de Investigación IVITA - El Mantaro es promisorio. En términos de consistencia, los valores de CCI son cercanos a excelentes y se compara favorablemente con los obtenidos por otros investigadores. Aun cuando en este estudio no se determinó la consistencia intraevaluador, los resultados sugieren que este índice muestra un componente razonable de objetividad.

Los análisis de correlación y regresión también se muestran favorables. Los valores de ICC correlacionan con todos los estima- dos de grasa corporal, pero particularmente bien con el IMC y la grasa total. El IMC es un estimado indirecto de la acumulación de grasa y, aunque su precisión ha sido cuestionada (Roubenoff et al., 1995), es usado extensivamente en diferentes especies. Entre los componentes de la grasa total, el cuerpo dorso-cervical-caudal es el que mejor correlaciona con los valores de ICC, posiblemente como resultado de la metodología de palpación. Las ecuaciones de regresión muestran coeficientes estadísticamente significativos con amplitudes que variaron entre $36 \mathrm{y}$ $42 \%$ del valor medio.

\section{Agradecimientos}

Esta investigación fue ejecutada gracias al financiamiento parcial del Vicerrectorado de Investigación de la UNMSM a través del proyecto CSI 080801031. Los autores agradecen la colaboración de estudiantes y técnicos de la EE IVITA El Mantaro en la obtención de los cuerpos grasos.

\section{Literatura Citada}

1. Aliaga L, Moncayo R, Rico E, Caycedo A. 2009. Producción de cuyes. Lima: Universidad Católica Sedes Sapientiae. 808 p.

2. Al-Sabbagh TA, Swanson LV, Thompson JM. 1995. The effect of ewe body condition at lambing on colostral immunoglobulin $\mathrm{G}$ concentration and lamb performance. J Anim Sci 73: 28602864.

3. Anitha A, Kapa SR, Jeepalyam S, Moorthy S, Rangappa P, Yemireddy KR. 2010. Development of the body condition score system in Murrah buffaloes: Validation through ultrasonic assessment of body fat reserves. J Vet Sci 11:1-8.

4. Audigé L, Wilson PR, Morris RS. 1998. A body condition score system and its use for farmed red deer hinds. New Zeal J Agr Res 41: 545-553. 
5. Domecq JJ, Skidmore AL, Lloyd JW, Kaneene JB. 1995. Validation of body condition scores with ultrasound measurements of subcutaneous fat of dairy cows. J Dairy Sci 78: 2308-2313.

6. Dorsten CM, Cooper DM. 2004. Use of body condition scoring to manage body weight in dogs. Contemp Top Lab Anim 43: 34-37.

7. Edmonson AJ, Lean IJ, Weaver LD, Farver T, Webster G. 1989. A body condition scoring chart of Holstein dairy cows. J. Dairy Sci 72: 68-78.

8. Esbenshade KL, Britt JH, Armstrong JD, Toelle VD, Stanislaw CM. 1986. Body condition of sows across parities and relationship to reproductive performance. J Anim Sci 62: 1187-1193.

9. Fitzgerald RF, Stalder KJ, Dixon PM, Johnson AK, Karriker LA, Jones GF. 2009. The accuracy and repeatability of sow body condition scoring. Prof Anim Sci 24: 415-425.

10. Fleiss JL, Shrout PE. 1978. Approximate interval estimation for a certain intraclass correlation coefficient. Psychometrika 43: 259-262.

11. Garnsworthy PC. 1988. The effect of energy reserves at calving on performance of dairy cows. In: Garnsworthy PC (ed). Nutrition and lactation in the dairy cow. England: Butterworths. p 157-170.

12. Gentry LR, Thompson DL Jr, Gentry GT Jr, Del Vecchio RP, Davis KA, Del Vecchio PM. 2004. The relationship between body condition score and ultrasonic fat measurement in mares of high versus low body condition. J Equine Vet Sci 24: 198-203.

13. Haneline M, Cooperstein R, Young M, Birkeland K. 2009. An annotated bibliography of spinal motion palpation reliability studies. J Can Chiropr Assoc 53: 40-58.

14. Hardin, R. 1990. Using body condition scoring in beef cattle management. The University of Georgia, College of Agricultural and Environmental Sciences, Cooperative Extension Service. Circular 817.
15. INCAGRO. 2006. Subproyecto "Desarrollo y evaluación de reproductores para maximizar la producción de cuyes de carne”. [Internet], [16 setiembre 2009]. Disponible en: http://www.incagro.gob.pe/ci-proyectos-faseii.shtml? $x=14593$

16. Jiménez R, Huamán R. 2010. Manual para el manejo de reproductores híbridos especializados en producción de carne. El Mantaro, Perú: INCAGROACRICUCEN-UNMSM. 175 p.

17. Kristensen E, Dueholm L, Vink D, Andersen JE, Jakobsen EB, IllumNielsen S, Petersen FA, Enevoldsen C. 2006. Within- and across-person uniformity of body condition scoring in Danish Holstein cattle. J Dairy Sci 89: 3721-3728.

18. Nicoll GB. 1979. Analysis of subjective body condition scores. Dunsinea Moorepark and Western Research Centres Research Report 1979. p 8-9. [Internet], [22 junio 2011]. Disponible en: http://www.cabdirect.org/abstracts/ 19810159018.html; jsessionid= 5624B9E57A97E11C73EDFF90F48B7DA3

19. Roche JR, Lee JM, McDonald KA, Berry DP. 2007. Relationships among body condition score, body weight, and milk production variables in pasturebased dairy cows. J Dairy Sci 90: 38023815.

20. Roubenoff R, Dallal GE, Wilson PWF. 1995. Predicting body fatness: The body mass index vs. estimation by bioelectrical impedance. Am J Public Health 85: 726728.

21. Sanson DW, West TR, Tatman WR, Riley ML, Judkins MB, Moss GE. 1993. Relationship of body composition of mature ewes with condition score and body weight. J Anim Sci 71: 1112-1116.

22. SAS Institute Inc. 2009. SAS/STAT ${ }^{\circledR}$ 9.2 User's Guide. $2^{\text {nd }}$ ed. Cary, NC: SAS Institute Inc. 7886 p.

23. Shrout PE, Fleiss JL. 1979. Intraclass correlations: Uses in assessing rater reliability. Psych Bull 86: 420-428. 
24. Shrubsole-Cockwill AN, Cockwill KRN, Parker DL. 2008. Omental torsion in a guinea pig (Cavia porcellus). Can Vet J 49: 898-900.

25. Veterinary Teaching Hospital, Michigan State University. 2011.
Feeding and caring for your guinea pig. College of Veterinary Medicine Menu Series. [Internet], [22 junio 2011]. Disponible en: http://old.cvm.msu.edu/ d o c u m e n t s / A b o o d _ P D F / GuineaPigs1.pdf 